The inception of pulsed discharges in air: simulations in background fields above and below breakdown

This content has been downloaded from IOPscience. Please scroll down to see the full text. 2014 J. Phys. D: Appl. Phys. 47445205

(http://iopscience.iop.org/0022-3727/47/44/445205)

View the table of contents for this issue, or go to the journal homepage for more

Download details:

IP Address: 131.155.2.68

This content was downloaded on 17/10/2014 at 09:45

Please note that terms and conditions apply. 


\title{
The inception of pulsed discharges in air: simulations in background fields above and below breakdown
}

\author{
Anbang Sun ${ }^{1}$, Jannis Teunissen ${ }^{1}$ and Ute Ebert ${ }^{1,2}$ \\ ${ }^{1}$ Centrum Wiskunde and Informatica (CWI), PO Box 94079, 1090 GB Amsterdam, The Netherlands \\ 2 Departments of Physics, Eindhoven University of Technology, 5612 AZ Eindhoven, The Netherlands \\ E-mail: absun1984@gmail.com
}

Received 10 June 2014, revised 25 August 2014

Accepted for publication 15 September 2014

Published 15 October 2014

\begin{abstract}
We investigate discharge inception in air, in uniform background electric fields above and below the breakdown threshold. We perform 3D particle simulations that include a natural level of background ionization in the form of positive and $\mathrm{O}_{2}^{-}$ions. In background fields below breakdown, we use a strongly ionized seed of electrons and positive ions to enhance the field locally. In the region of enhanced field, we observe the growth of positive streamers, as in previous simulations with $2 \mathrm{D}$ plasma fluid models. The inclusion of background ionization has little effect in this case. When the background field is above the breakdown threshold, the situation is very different. Electrons can then detach from $\mathrm{O}_{2}^{-}$and start ionization avalanches in the whole volume. These avalanches together create one extended discharge, in contrast to the 'double-headed' streamers found in many fluid simulations.
\end{abstract}

Keywords: discharge, electrical breakdown, breakdown field, background ionization, streamer, overvolted

(Some figures may appear in colour only in the online journal)

\section{Introduction}

Developments in pulsed power technology have increased the interest in pulsed discharges over the last two decades. These discharges now have a wide range of applications, for example, ozone generation [1-4], gas and water cleaning $[2,5,6]$, flow control and plasma assisted ignition and combustion [7]. Pulsed discharges appear also in thunderstorms and in high voltage technology for electricity networks.

Here, we focus on the initial development of such pulsed discharges in air at standard temperature and pressure, which we study with a three-dimensional (3D) particle model. We consider two different cases for the background electric field: it is either only locally above the breakdown threshold, or globally. Homogeneous background fields above breakdown can occur for example between parallel electrodes, or far from charge accumulations, as in thunderclouds $[8,9]$. The main objective of the current paper is to show that in air one needs to distinguish between fields above and below breakdown, due to the presence of background ionization.
If the background field is only locally above breakdown, a discharge can only grow in that region, typically forming a streamer. Streamers are fast growing plasma filaments that can penetrate into non-ionized regions due the electric field enhancement at their tips. They have been studied in different gases and in different electric field configurations both experimentally [10-16] and numerically [17-26].

If the background field is globally above breakdown, ionization processes can take place in the whole volume, at least if some background ionization is present to provide the first free electrons. Because most background ionization is present in the form of negative ions, this first requires electron detachment, which we discuss in some detail. We will see that the growth of electron avalanches in the whole volume can actually inhibit the formation of separate streamers.

The outline of the paper is as follows. In section 2, we first briefly discuss previous work. In section 3 , we introduce the simulation model and discuss background ionization and electron detachment. In section 4 , we present simulation results 
showing streamer formation in fields only locally above breakdown. These results are in qualitative agreement with previous work. Then, in section 5, we investigate discharge formation in background electric fields globally above breakdown. The results here show that the presence of background ionization leads to the formation of a 'global discharge', consisting of many electron avalanches.

\section{Previous work}

Up to now, pulsed discharges in air have mainly been simulated with plasma fluid models [21, 27-32], where the charged particles are approximated by densities. The most common fluid model assumes that the electrons drift, diffuse and react (ionize), with the coefficients for these processes determined by the local electric field strength. Typically cylindrical symmetry is assumed and therefore these fluid models need just two spatial coordinates, making them computationally much less expensive than our 3D particle code. Authors typically place some localized initial ionization in the domain to start a discharge [21, 3032]. In background fields above the breakdown threshold, this ionization seed then develops into a 'double-headed' streamer. The effect of including natural background ionization (and detachment) has not been studied with these models.

In [33], we have recently demonstrated that including background ionization and detachment can be important for discharges in air above breakdown. We therefore used the same simulation model to compare discharge formation in atmospheric air with and without 'natural' background ionization. The present paper is an extension of [33]. The main differences are that we can discuss the simulation model and the results in more detail here and that we include results in fields only locally above breakdown.

\section{The set-up of the MC particle model}

In recent years, we have developed a $3 \mathrm{D}$ particle code of the PIC-MCC (particle-in-cell, Monte-Carlo collision) type [34] to study discharge inception. This simulation model is made available on our group's webpage [35]. The reason for using a $3 \mathrm{D}$ particle model is that the start of a discharge is often a stochastic process, that lacks cylindrical (or other) symmetry. In the model, electrons are tracked as particles. Ions are assumed to be immobile and are included as densities. They only contribute to space charge effects. Neutral gas molecules provide a background that electrons can randomly collide with; they are included in the code as a random background of given density.

The simulations of the present paper are performed in dry air $\left(80 \% \mathrm{~N}_{2}, 20 \% \mathrm{O}_{2}\right)$ at 1 bar and 293 Kelvin. For the electrons, we include elastic, excitation, ionization and attachment collisions with the neutral gas molecules. We use the cross sections from the SIGLO database [36] and the null-collision method to select collisions [37], with isotropic scattering after every collision. We ignore electron-electron and electron-ion collisions, because the degree of ionization in a pulsed discharge in STP air is typically below $10^{-4}$, which is also the case in the simulations we perform.
Simulating a discharge with a 3D particle code is computationally expensive, especially as the discharge grows. This limits the simulations we can perform to the first nanoseconds of a discharge, during which the inception takes place. On this time scale, heating, recombination and multi-step excitation or ionization can be neglected.

\subsection{Adaptive particle management}

As the number of electrons in a typical discharge quickly rises to $10^{8}$ or more, so-called super-particles have to be used. Using super-particles with a fixed weight would induce significant stochastic errors and therefore we employ 'adaptive particle management' as described in [38]. The weight of simulated particles can then be adjusted by merging or splitting them and care is taken to not alter their properties in a systematic way. A particle $i$ can only be merged with its closest neighbor $j$ that also needs to be merged, with 'closest' defined as minimizing

$$
d^{2}=\left(\vec{x}_{i}-\vec{x}_{j}\right)^{2}+\lambda^{2}\left|v_{i}-v_{j}\right|^{2},
$$

where $\vec{x}$ denotes the Cartesian position vector, $v$ is the norm of the velocity and $\lambda$ is a scaling factor that we set to one picosecond. A newly formed merged particle gets its velocity at random from one of the original particles, while its position is set to the weighted average position, see [38] for a comparison of different schemes to merge particles. We adjust the weights so that every cell of the grid (see below) contains at least 50 simulation particles. So if no more than 50 electrons are present in a cell, then each simulation particle represents a single electron. But where the electron density is high, with much more than 50 electrons in a cell, most simulation particles represent many electrons.

\subsection{Adaptive mesh refinement for the electric field}

In the particle code, the electric field is computed from the electric potential. The potential is computed by solving Poisson's equation with the charge density as the source term, using the HW3CRT solver from the FISHPACK library [39]. When space charge effects become important in a discharge, a grid fine enough to resolve the space charge structures has to be used. In our simulations, we use the following criterion for the grid spacing

$$
\Delta x<1 / \alpha(E),
$$

where $\alpha(E)$ is the ionization coefficient, that describes the average number of ionizations a single electron will generate per unit length in a field of strength $E$. For air at 1 bar and in an electric field of $15 \mathrm{MV} \mathrm{m}^{-1}$, a typical field for streamer tips, this gives $\Delta x \sim 5 \mu \mathrm{m}$. Because a typical simulation domain measures at least a few $\mathrm{mm}$ in each direction, using such a fine grid everywhere is computationally infeasible. Therefore, we have implemented block-based adaptive mesh refinement, in the same way as in [40], although now in 3D. First, the electric potential is computed on a uniform, coarse grid. Then the rectangular area that contains the points at which the electric field 
is larger than some threshold is refined, by a factor of two. The electric potential in the refined rectangle is then computed by imposing Dirichlet boundary conditions interpolated from the coarse grid. This procedure is repeated with the refinement criterion given by equation (2).

For the simulation of streamer discharges, the block-based grid refinement strategy described above works relatively well, because high electric fields are present only in a small region. But for the simulation of discharges that spread out over the whole domain, as we will see in section 5, this type of grid refinement does not reduce the computational cost much.

\subsection{Photoionization}

Photoionization provides a non-local ionization mechanism in air. This is especially important for the propagation of positive streamers, that need a source of free electrons ahead of them to propagate. We use the same approach as in [41, 42], where a discrete, stochastic version of Zhelezniak's photoionization model [43] is implemented. In this model, the average density of ionizing photons $S_{\text {ph }}$ produced at $\vec{r}$ is given by

$$
S_{\mathrm{ph}}(\vec{r})=S_{\text {ion }}(\vec{r}) \eta(E),
$$

where $S_{\text {ion }}$ represents the number of ionizations and $\eta(E)$ is an efficiency, estimated from experimental measurements, that depends on the local electric field $E$ and the gas mixture. When an ionizing photon is generated, its place of absorption is determined using random numbers and at that position an electron-ion pair is created. The average absorption distance is about $0.5 \mathrm{~mm}$ in air at 1 bar. For details about the implementation of the photoionization model we refer to [41].

\subsection{Electron detachment from background ionization}

In atmospheric air, there is always some background ionization present, due to radioactivity and cosmic or solar radiation. Previous discharges can also play a role, both in nature [44] and in the lab [11]. At standard temperature and pressure, the free electrons that are created by these sources attach to oxygen molecules mostly by three-body attachment [45]:

$$
\begin{gathered}
\mathrm{e}+\mathrm{O}_{2}+\mathrm{O}_{2} \rightarrow \mathrm{O}_{2}^{-}+\mathrm{O}_{2}, \\
\mathrm{e}+\mathrm{N}_{2}+\mathrm{O}_{2} \rightarrow \mathrm{O}_{2}^{-}+\mathrm{N}_{2} .
\end{gathered}
$$

These negative ions have a longer life time than the electrons. Inside buildings, background ionization levels of $10^{3}-10^{4} \mathrm{~cm}^{-3}$ are typical, primarily due to the decay of radon, see [46] for a review. When $\mathrm{O}_{2}^{-}$molecules collide with a neutral gas molecule, the attached electrons can detach again, so that reactions (4) and (5) are reversed:

$$
\begin{aligned}
& \mathrm{O}_{2}^{-}+\mathrm{O}_{2} \rightarrow \mathrm{e}+\mathrm{O}_{2}+\mathrm{O}_{2}, \\
& \mathrm{O}_{2}^{-}+\mathrm{N}_{2} \rightarrow \mathrm{e}+\mathrm{N}_{2}+\mathrm{O}_{2} .
\end{aligned}
$$

We use the detachment rates given in [45] (reactions (56) and (57) in that reference):

$$
k_{6}=2.7 \cdot 10^{-10} \sqrt{T / 300} \exp (-5590 / T) \mathrm{cm}^{3} \mathrm{~s}^{-1},
$$

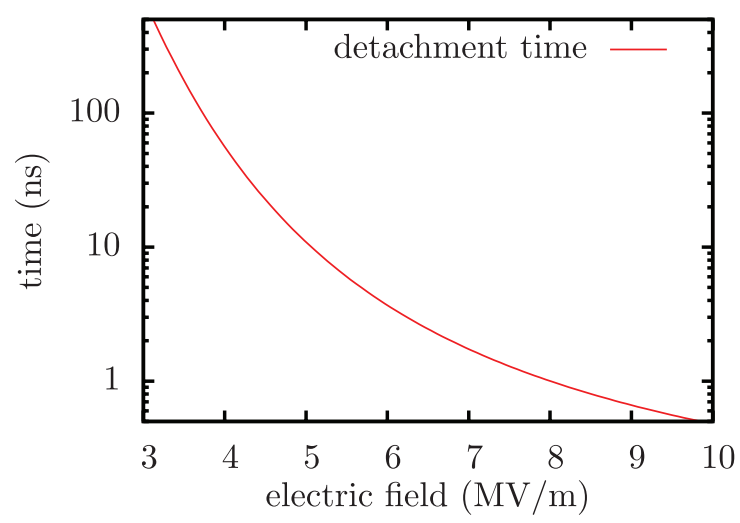

Figure 1. The detachment time $\tau_{D}$ as a function of the electric field strength in STP air. In higher fields, negative ions have a higher energy and drift faster, so they are more likely to lose an electron in a collision with a neutral molecule.

$$
k_{7}=1.9 \cdot 10^{-12} \sqrt{T / 300} \exp (-4990 / T) \mathrm{cm}^{3} \mathrm{~s}^{-1},
$$

where $T$ is the gas temperature in Kelvin, in the absence of an electric field. In our case, there is an applied electric field, which means that the ions will have a higher effective temperature $T_{\text {ion }}$ than the background gas. It was suggested to us [47] to take $T$ as the average of the gas and the ion temperature,

$$
T=\left(T_{\text {gas }}+T_{\text {ion }}\right) / 2,
$$

with the latter given by

$T_{\text {ion }}=T_{\text {gas }}+\frac{2}{3 k_{\mathrm{B}}} M_{\text {ion }} v_{\text {ion }}^{2}=T_{\text {gas }}+\frac{2}{3 k_{\mathrm{B}}} M_{\text {ion }}\left(\mu_{\text {ion }} E\right)^{2}$,

where $k_{\mathrm{B}}$ is the Boltzmann constant, $M_{\text {ion }}$ the ion mass, $v_{\text {ion }}$ the ion drift velocity and $\mu_{\text {ion }}$ the ion mobility, which we approximate by $\mu_{\text {ion }}=2 \cdot 10^{-4} \mathrm{~m}^{2}(\mathrm{Vs})^{-1}$. Note that it is assumed that the total energy of the ion is twice the 'drift energy' $\left(M_{\text {ion }} v_{\text {ion }}^{2} / 2\right)$ [48].

Using equations (6)-(11), we can compute the total rate at which electrons detach from $\mathrm{O}_{2}^{-}$ions in a given electric field $E$. We call this rate the detachment rate and its inverse the detachment time $\tau_{D}$. In figure 1 , the dependence of $\tau_{D}$ on the electric field strength is shown. At the breakdown field $\left(3 \mathrm{MV} \mathrm{m}^{-1}\right)$ the detachment time is about $500 \mathrm{~ns}$, but at $5 \mathrm{MV} \mathrm{m}^{-1}$ it is only $10 \mathrm{~ns}$.

We currently consider only $\mathrm{O}_{2}^{-}$ions for detachment, although $\mathrm{O}^{-}$can also form due to dissociative attachment, mostly at lower pressures or higher electron energies. From these ions electrons can detach in fields much below breakdown [49-51]. Furthermore, many other types of ions can be generated by chemical reactions $[50,52]$.

We want to emphasize that both detachment and photoionization are characteristic for nitrogen/oxygen mixtures. In pure gases or other mixtures these processes might be absent or much weaker, see e.g. [10].

\section{Discharges in background fields below breakdown}

In this section, we show an example of discharge formation in background electric fields below breakdown. Of course, the 


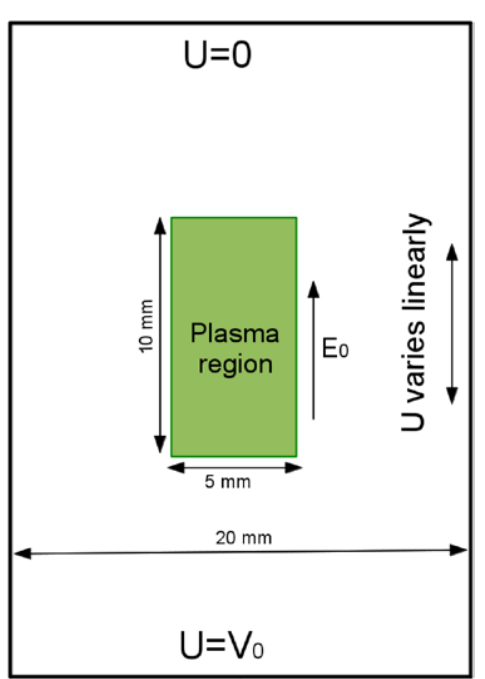

Figure 2. Schematic view of the computational domain in the undervolted simulations. The simulated plasma region is embedded in a four times larger domain.

field has to exceed the breakdown threshold in some region, otherwise a discharge cannot start. Such a region can be created by sharp electrodes or by polarizable object such as dust, water droplets or ice crystals. We use a method that has been commonly used in fluid simulations of streamers for the past 30 years, namely to place an ionized seed in the domain [21, 30-32]. The electrons in such a seed move in the background field, polarizing the seed, so that the electric field gets enhanced at the endpoints. Our results are in agreements with previous modeling efforts and the inclusion of background ionization has little influence.

\subsection{Conditions for the simulations below breakdown}

The computational domain that we use for fields below breakdown is shown in figure 2. It contains two parts: an interior grid of $5 \times 5 \times 10 \mathrm{~mm}^{3}$, in which we use the particle model and a four times larger grid around it that is used to set the boundary conditions for the electric potential on the interior grid. Dirichlet boundary conditions are imposed on the sides of the larger grid to get a homogeneous background field $E_{0}<E_{k}$ in the vertical direction. Inside the interior grid we use adaptive mesh refinement, so that the strong electric fields around streamer heads can be resolved. As a background gas we use dry air at 1 bar and 293 Kelvin, with a density of $10^{4}$ $\mathrm{O}_{2}^{-}$ions per $\mathrm{cm}^{3}$ and an equal density of positive ions.

\subsection{Results}

We use is a long, neutral ionized column, similar to the initial condition used in [53], but then scaled to ground pressure. The peak ion and electron density is $1.3 \times 10^{13} \mathrm{~cm}^{-3}$. In the two lateral directions, the distribution of electrons and ions is Gaussian, with a width of $0.2 \mathrm{~mm}$. The distribution of plasma in the vertical direction is uniform over a length of $4 \mathrm{~mm}$; at the endpoints there is again a Gaussian distribution. An external electric field of $\sim 0.5 E_{k}$ is applied in the vertical direction.

Figure 3 shows how this seed develops further in the simulations. First, the column gets polarized and negative and positive charge layers emerge at the top and bottom of the column, respectively. After $\sim 10 \mathrm{~ns}$, a positive streamer forms at the upper end of the column, as shown in the first row of figure 3. At the lower end, electrons spread out or attach to neutral molecules. On the time scales that can be simulated with our particle model, we have not observed negative streamers emerging. An important difference between positive and negative streamers is that positive streamers grow from electrons drifting inwards towards their head, while negative streamers grow from the electrons drifting outwards. Thus, the space charge layer of a positive streamer head is formed by rather immobile ions, while the space charge layer of a negative streamer head is formed by mobile electrons. Negative streamers are therefore typically wider and more diffusive, with less field enhancement and they do not form as easily [21].

\subsection{Effect of background ionization}

We have repeated the simulations presented above without initial background ionization and the results showed no apparent differences. There are two reasons for this. First, photoionization produces most free electrons ahead of the front after the discharge has started [17]. Second, detachment from $\mathrm{O}_{2}^{-}$ only plays a role in the region above breakdown, but electrons are already present there due to the initial seed. In regions below breakdown, the detachment time is more than $500 \mathrm{~ns}$ (see figure 1) and if an electron would appear, then it would not produce an ionization avalanche.

The simulation results look qualitatively similar to those obtained with typical 2D fluid simulations. This will be different in the simulations above breakdown presented in the next section.

\section{Discharges in background fields above breakdown}

In this section, we present simulations in a background field globally above breakdown. Below, we first describe the computational domain used for these simulations.

\subsection{Simulation conditions}

In this section, we present new simulation results for a discharge developing in a field of $6 \mathrm{MV} \mathrm{m}^{-1}$, twice the breakdown field. The same level of background ionization is present as in section 4 , namely a density of $10^{4} \mathrm{~cm}^{-3} \mathrm{O}_{2}^{-}$and positive ions. However, we use a different computational domain here, because we want to simulate the development of a discharge that is not in contact with physical boundaries, like electrodes. Therefore, we use periodic boundary conditions in the $x$ and $y$ direction, while limiting the region where background ionization is present in the $z$ direction. In other words, we simulate the development of a thick discharge layer growing from 


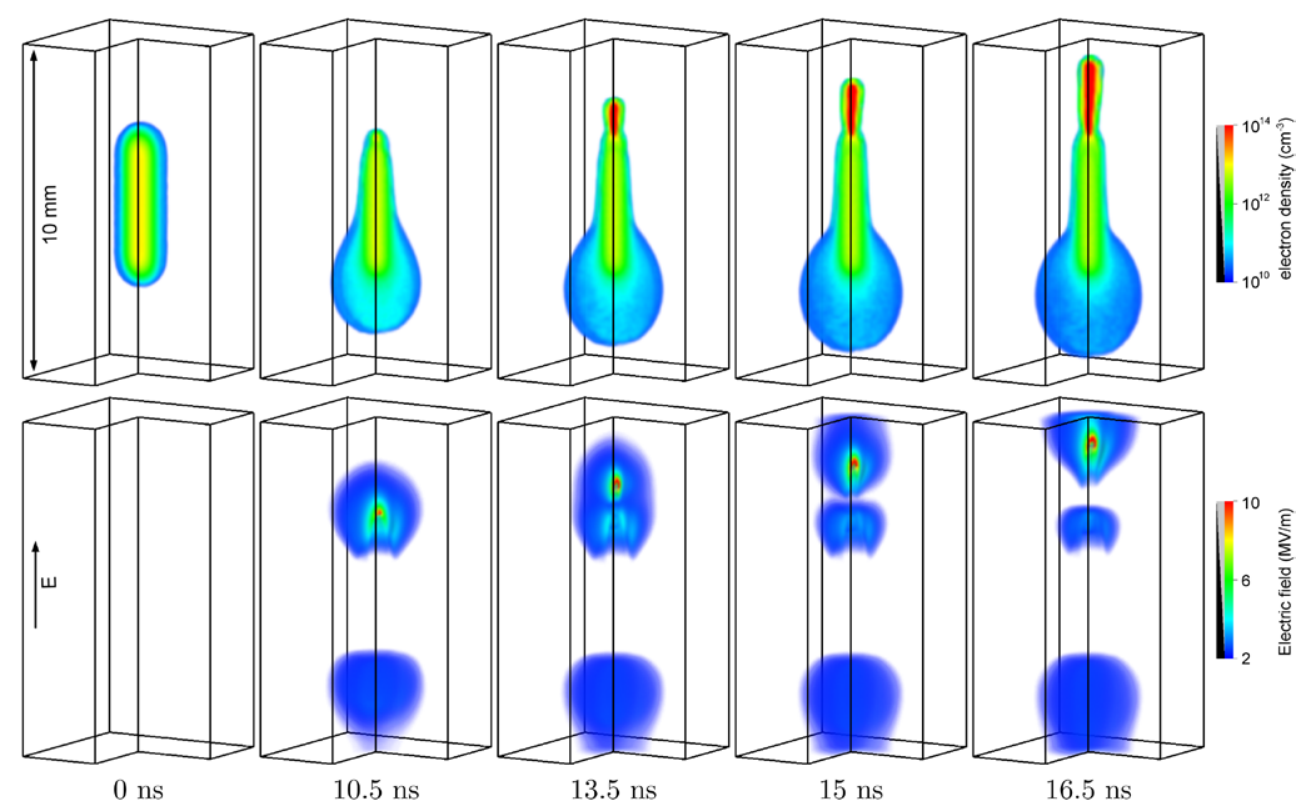

Figure 3. Simulation results in a field of $1.7 \mathrm{MV} \mathrm{m}^{-1}$, about half the breakdown value. The top row shows the electron density and the bottom row the electric field, at various times. Initially an ionized column is present, that causes local field enhancement. The domain measures $10 \times(5 \mathrm{~mm})^{2}$ and is cut open. The figures were generated using volume rendering; opacity is indicated in the colorbar.

background ionization. The elongated computational domain is shown in figure 4 , where the region with background ionization is shaded green. At the top and bottom of the domain we apply Neumann boundary conditions for the electric potential, thereby creating a uniform background field $E_{0}$ of $6 \mathrm{MV} \mathrm{m}^{-1}$. We remark that in the GRL [33] we were less careful with the boundary conditions and used something similar to figure 2 .

We do not use grid refinement to calculate the generated electric field in this simulation, as grid refinement would be required nearly everywhere in the pre-ionized region. The static grid contains $100 \times 100 \times 535$ cells, with a cell length of $15 \mu \mathrm{m}$. The domain length is chosen in such a manner that the discharge does not reach its boundaries within the time simulated.

\subsection{Simulated discharge evolution}

Figure 5 shows the evolution of the electron density and the electric field in four time steps between $4.5 \mathrm{~ns}$ and $5.4 \mathrm{~ns}$. The evolution of the discharge can be characterized as follows. First, free electrons appear due to detachment. As can be seen in figure 1 , the characteristic detachment time in a field of $6 \mathrm{MV} \mathrm{m}^{-1}$ is about $3 \mathrm{~ns}$. Then these free electrons start electron avalanches, that quickly grow due to impact ionization. The growing avalanches also produce photoionization, thereby starting additional avalanches. Eventually, many avalanches emerge in the simulation domain.

After about $5 \mathrm{~ns}$, space charge effects start to become important, causing the electric field to increase locally up to $\sim 9 \mathrm{MV} \mathrm{m}^{-1}$ while decreasing elsewhere. These space charge effects increase in magnitude until the simulation stops at $5.4 \mathrm{~ns}$. The distribution of the electric field values is shown in figure 6. After $4.5 \mathrm{~ns}$, almost the complete system is still at the background field of $6 \mathrm{MV} \mathrm{m}^{-1}$, but after $5.4 \mathrm{~ns}$, about $8 \%$ of the volume has a field lower than the breakdown value of $3 \mathrm{MV} \mathrm{m}^{-1}$

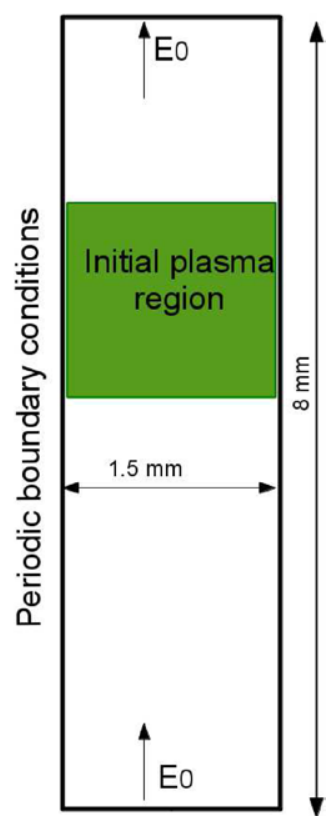

Figure 4. Schematic view of the computational domain in the 3D overvolted simulations. In the vertical direction, the electric field is fixed to a value $E_{0}$ of $6 \mathrm{MV} \mathrm{m}^{-1}$. Periodic boundary conditions are used in the two lateral directions. Initially background ionization is present in the green region.

Figure 7 shows the distribution of electric fields in the simulation in another manner; it shows the electric field averaged over the horizontal planes intersecting figure 5 and plotted as a function of the longitudinal coordinate. The screening of the electric field occurs in a 'noisy' way and the electric field varies significantly inside the discharge. This is not so surprising, as initially only about 45 negative ions $\left(\mathrm{O}_{2}^{-}\right)$are present. With these ions randomly placed in a volume of $4.5 \mathrm{~mm}^{3}$, we do not expect a discharge homogeneously filled with ionization. 


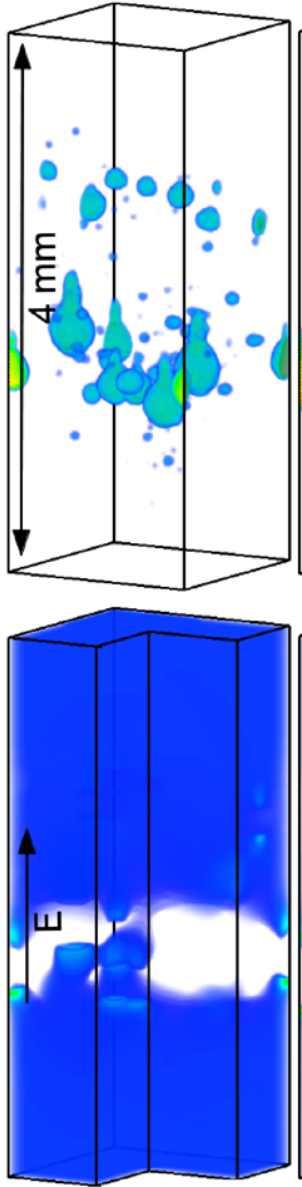

$4.5 \mathrm{~ns}$

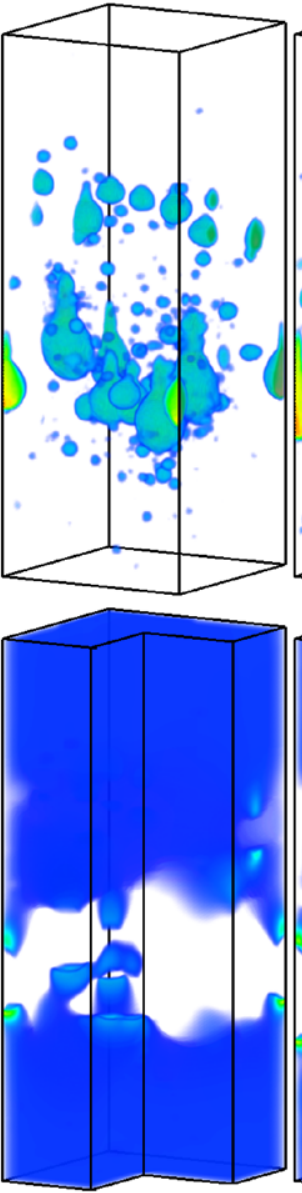

$4.8 \mathrm{~ns}$

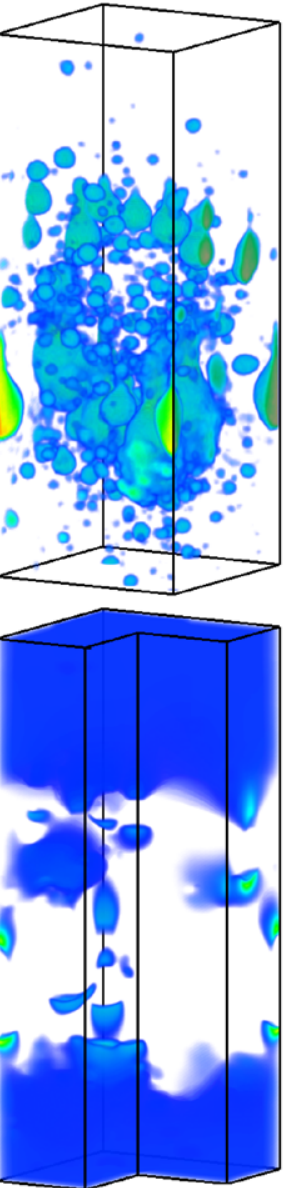

$5.1 \mathrm{~ns}$

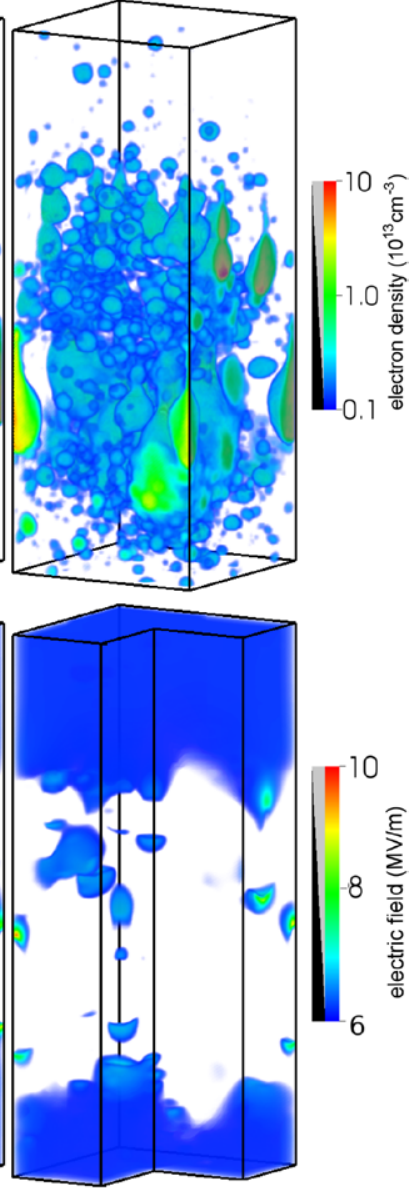

$5.4 \mathrm{~ns}$

Figure 5. The time evolution of the electron density (top row) and of the electric field (bottom row). Background ionization is initially present in the green region of figure 4, in the form of $\mathrm{O}_{2}^{-}$and positive ions, both with a density of $10^{4} \mathrm{~cm}^{-3}$. The gas is dry air at 1 bar and $293 \mathrm{~K}$ in an upward directed homogeneous electric field of $6 \mathrm{MV} \mathrm{m}^{-1}$, which is about two times the breakdown field. The domain between $2 \mathrm{~mm}$ and $6 \mathrm{~mm}$ in the vertical direction of figure 4 is shown.

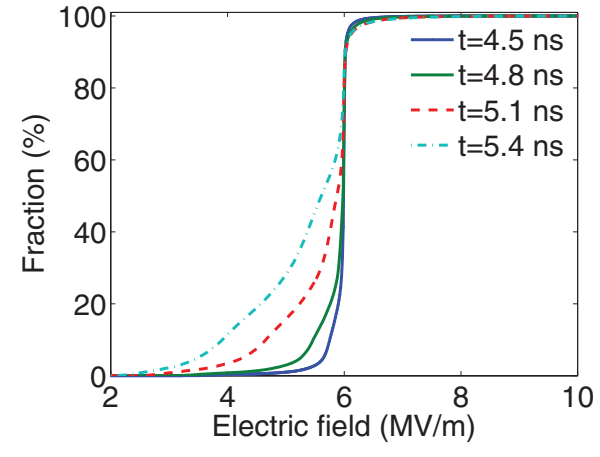

Figure 6. The volume fraction with a field smaller than $E$ as a function of $E$, for the simulation shown in figure 5. We evaluate the green domain shown in figure 4.

The simulation stops when there are too many simulation particles for the computer's memory, which happened here at about $3 \cdot 10^{7}$ particles.

\subsection{Effect of background ionization}

Above breakdown, we observe a 'global' discharge. Free electrons can appear anywhere in the region containing

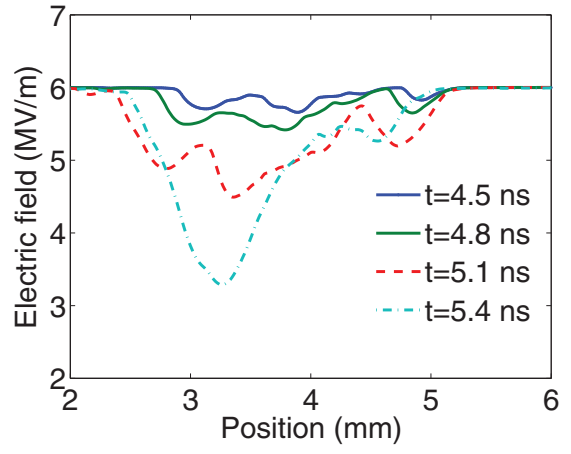

Figure 7. The averaged electric field of the simulation shown in figure 5 . The averaging is performed over planes perpendicular to the background field, see figure 4 .

pre-ionization, due to detachment from $\mathrm{O}_{2}^{-}$ions. The electrons then form electron avalanches, building up space charge. The avalanches together reduce the field in the interior of the discharge, which can clearly be seen in figure 5. There seems to be competition between local streamer formation and homogeneous breakdown: on the one hand, the collective space charge from all the avalanches inhibits the formation of streamers, which are characterized by strong local field 
enhancement. On the other hand, there is actually some local field enhancement due to the limited number of avalanches, which makes the discharge rather inhomogeneous.

Our results are very different from previous publications in which 'double-headed' streamers were observed in background fields above breakdown, see for example [26, 28, 29, 41]. Therefore, we think that the inclusion of background ionization (and detachment reactions) is essential for discharge simulations in air above the breakdown threshold. Note that we would observe a similar discharge if we had included a density of free electrons, or other ions from which electrons can detach, instead of $\mathrm{O}_{2}^{-}$. The main difference with previous publications is caused by the fact that the pre-ionization is distributed uniformly over the domain, instead of locally into a single seed.

Note the difference between the simulations we have shown below and above breakdown: below breakdown, a localized seed was required to start a streamer discharge, while above breakdown, homogeneous pre-ionization prevents the (immediate) formation of streamers.

\subsection{Homogeneous breakdown}

If we increase the amount of pre-ionization in the simulations, the discharges will become more homogeneous and form a layer, see [54] for a related experimental example. Homogeneous, high-pressure discharges have been generated for use in gas combustion and excimer lasers, see the review in [55]. For the excimer lasers it is important to prevent arc formation and therefore the discharge should be as homogeneous as possible. Typically, a high level of pre-photoionization is generated for this purpose. There have been several studies estimating the required initial ionization density $n_{0}$ to prevent streamer or arc formation [56-58]. These estimates are typically derived by assuming that avalanches should overlap (to some degree) when space charge effects become important. In a recent publication [59], we have also given such an estimate

$$
n_{0} \approx \frac{1}{216 k^{3}}\left(\frac{v_{d} \alpha_{\mathrm{eff}}}{D_{e}}\right)^{3 / 2}
$$

where $k$ is a number around one, $v_{d}$ the electron drift velocity, $\alpha_{\text {eff }}$ the effective ionization coefficient and $D_{e}$ the electron diffusion coefficient.

In figure 5 we could already see that the electric field in the interior in the discharge is reduced in time. In [59], we have presented an estimate for the 'ionization screening time', which is the time it takes for the interior field to drop below breakdown

$$
\tau_{\text {is }}=\ln \left(1+\frac{\alpha_{\mathrm{eff}} \varepsilon_{0} E_{0}}{e n_{0}}\right) /\left(\alpha_{\mathrm{eff}} v_{d}\right)
$$

where $E_{0}$ is the applied field and $n_{0}$ the initial electron density. If there are instead of electrons negative ions, there will be a delay in the screening process because detachment takes some time. This delay can be approximated by [59]

$$
\tau_{\text {delay }}=\ln \left(1+\tau_{d} \alpha_{\text {eff }} v_{d}\right) /\left(\alpha_{\text {eff }} v_{d}\right),
$$

where $\tau_{d}$ is the detachment time.

\section{Conclusion}

We have studied pulsed discharge formation in electric fields above and below the breakdown threshold with a 3D particle model for air at standard temperature and pressure. Photoionization, a natural level of $10^{4} \mathrm{~cm}^{-3} \mathrm{O}_{2}^{-}$ions due to background ionization and electron detachment were included.

In background electric fields below the breakdown value, we observed the formation of a positive streamer if the field is locally sufficiently enhanced. The inclusion of background ionization did not affect the results and our 3D particle model gives similar results as commonly used 2D plasma fluid models. But in background electric fields above breakdown, we see discharges distributed over the whole domain instead of the 'double-headed' streamers often appearing in other publications. The major cause for this difference is the inclusion of homogeneous background ionization. Free electrons appear at many different places due to detachment from $\mathrm{O}_{2}^{-}$ions and start electron avalanches. These avalanches interact and overlap and can eventually screen the electric field in the interior of the discharge, which we discuss in a separate paper [59].

The main conclusion of our work is therefore that in background fields above breakdown, the distribution of the pre-ionization determines the evolution of the discharge. Therefore relevant sources of background ionization should be included.

\section{Acknowledgments}

ABS acknowledges the support by an NWO Valorization project at CWI and by STW projects 10118 and 12119. JT was supported by STW project 10755 .

\section{References}

[1] Samaranayake W J M, Miyahara Y, Namihira T, Katsuki S, Sakugawa T, Hackam R and Akiyama H 2000 Pulsed streamer discharge characteristics of ozone production in dry air IEEE Trans. Dielectr. Electr. Insul. 7 254-260

[2] van Veldhuizen E M 2000 Electrical Discharges for Environmental Purposes: Fundamentals and Applications (New York: Nova Science)

[3] van Heesch E J M, Winands G J J and Pemen A J M 2008 Evaluation of pulsed streamer corona experiments to determine the O* radical yield J. Phys. D: Appl. Phys. 41234015

[4] Ono R and Oda T 2006 Ozone production process in pulsed positive dielectric barrier discharge J. Phys. D: Appl. Phys. 40 176-82

[5] Grabowski L R, van Veldhuizen E M, Pemen A J M and Rutgers W R 2006 Corona above water reactor for systematic study of aqueous phenol degradation Plasma Chem. Plasma Process. 26317

[6] Winands G J J, Yan K, Pemen A J M, Nair S A, Liu Z and Van Heesch E J M 2006 An industrial streamer corona plasma system for gas cleaning IEEE Trans. Plasma Sci. 34 2426-33

[7] Starikovskaia S M 2014 Plasma-assisted ignition and combustion: nanosecond discharges and development of kinetic mechanisms J. Phys. D: Appl. Phys. 47353001 
[8] Gurevich A V and Karashtin A N 2013 Runaway breakdown and hydrometeors in lightning initiation Phys. Rev. Lett. 110185005

[9] Li J and Cummer S 2012 Relationship between sprite streamer behavior and lightning-driven electric fields J. Geophys. Res. 117 A01317

[10] Nijdam S, van de Wetering F M J H, Blanc R, van Veldhuizen E M and Ebert U 2010 Probing photoionization: experiments on positive streamers in pure gases and mixtures J. Phys. D: Appl. Phys. 43145204

[11] Nijdam S, Wormeester G, van Veldhuizen E M and Ebert U 2011 Probing background ionization: positive streamers with varying pulse repetition rate and with a radioactive admixture J. Phys. D: Appl. Phys. 44455201

[12] van Veldhuizen E M and Rutgers W R 2002 Pulsed positive corona streamer propagation and branching J. Phys. D: Appl. Phys. 35 2169-79

[13] van Veldhuizen E M and Rutgers W R 2003 Inception behaviour of pulsed positive corona in several gases J. Phys. D: Appl. Phys. 36 2692-6

[14] Yi W J and Williams P F 2002 Experimental study of streamers in pure $\mathrm{N}_{2}$ and $\mathrm{N}_{2} / \mathrm{O}_{2}$ mixtures and a $13 \mathrm{~cm}$ gap J. Phys. D: Appl. Phys. 35 205-218

[15] Dubrovin D, Nijdam S, van Veldhuizen E M, Ebert U, Yair Y and Price C 2010 Sprite discharges on Venus and Jupiterlike planets: a laboratory investigation J. Geophys. Res. 115 A00E34

[16] Hegeler F and Akiyama H 1997 Spatial and temporal distributions of ozone after a wire-to-plate streamer discharge IEEE Trans. Plasma Sci. 25 1158-65

[17] Wormeester G, Pancheshnyi S, Luque A, Nijdam S and Ebert U 2010 Probing photo-ionization: simulations of positive streamers in varying $\mathrm{N}_{2}: \mathrm{O}_{2}$-mixtures $J$. Phys. $D$ : Appl. Phys. 43505201

[18] Celestin S, Bonaventura Z, Zeghondy B, Bourdon A and Sgur P 2009 The use of the ghost fluid method for Poissons equation to simulate streamer propagation in point-to-plane and point-to-point geometries J. Phys. D: Appl. Phys. 42065203

[19] Tholin F and Bourdon A 2013 Simulation of the stable quasiperiodic glow regime of a nanosecond repetitively pulsed discharge in air at atmospheric pressure Plasma Sources Sci. Technol. 22045014

[20] Yair Y, Takahashi Y, Yaniv R, Ebert U and Goto Y 2009 A study of the possibility of sprites in the atmospheres of other planets J. Geophys. Res. 114 E09002

[21] Luque A, Ratushnaya V and Ebert U 2008 Positive and negative streamers in ambient air: modelling evolution and velocities J. Phys. D: Appl. Phys. 41234005

[22] Luque A, Ebert U and Hundsdorfer W 2008 Interaction of streamer discharges in air and other oxygen-nitrogen mixtures Phys. Rev. Lett. 101075005

[23] Aleksandrov N L and Bazelyan E M 1996 Simulation of long-streamer propagation in air at atmospheric pressure J. Phys. D: Appl. Phys. 29 740-52

[24] Naidis G V 2012 Simulation of a single streamer traveling along two counterpropagating helium jets in ambient air IEEE Trans. Plasma Sci. 40 2866-9

[25] Babaeva N Yu and Naidis G V 1998 2D modeling of positive streamer propagation in flue gases in sphere-plane gaps IEEE Trans. Plasma Sci. 264145

[26] Dhali S and Williams P 1985 Numerical simulation of streamer propagation in nitrogen at atmospheric pressure Phys. Rev. A 31 1219-21

[27] Pasko V P, Inan U S and Bell T F 1998 Spatial structure of sprites Geophys. Res. Lett. 25 2123-6

[28] Liu N 2004 Effects of photoionization on propagation and branching of positive and negative streamers in sprites J. Geophys. Res. 109 A04301
[29] Qin J, Celestin S and Pasko V P 2012 Formation of single and double-headed streamers in sprite-halo events Geophys. Res. Lett. 39 L05810

[30] Pasko V P 2007 Red sprite discharges in the atmosphere at high altitude: the molecular physics and the similarity with laboratory discharges Plasma Sources Sci. Technol. $16 \mathrm{~S} 13 \mathrm{~S} 29$

[31] Bourdon A, Pasko V P, Liu N Y, Clestin S, Sgur P and Marode E 2007 Efficient models for photoionization produced by non-thermal gas discharges in air based on radiative transfer and the Helmholtz equations Plasma Sources Sci. Technol. 16656678

[32] Bessires D, Paillol J, Bourdon A, Sgur P and Marode E 2007 A new 1D moving mesh method applied to the simulation of streamer discharges J. Phys. D: Appl. Phys. 40 6559-70

[33] Sun A B, Teunissen J and Ebert U 2013 Why isolated streamer discharges hardly exist above the breakdown field in atmospheric air Geophys. Res. Lett. 40 2417-22

[34] Birdsall C K and Langdon A B 1991 Plasma Physics Via Computer Simulation (Bristol: Institute of Physics)

[35] Multiscale Plasma Modeling 2014 www.cwimd.nl

[36] Pitchford L C and Boeuf J P 2014 The siglo database www.lxcat.net

[37] Nanbu K 2000 Probability theory of electron-molecule, ionmolecule, molecule-molecule and Coulomb collisions for particle modeling of materials processing plasmas and cases IEEE Trans. Plasma Sci. 28971990

[38] Teunissen J and Ebert U 2014 Controlling the weights of simulation particles: adaptive particle management using k-d trees J. Comput. Phys. 259318330

[39] Adams J P, Swarztrauber P N and Sweet R 2011 FISHPACK90 www.cisl.ucar.edu/css/software/fishpack90/

[40] Montijn C, Hundsdorfer W and Ebert U 2006 An adaptive grid refinement strategy for the simulation of negative streamers J. Comput. Phys. 219801835

[41] Chanrion O and Neubert T 2008 A PIC-MCC code for simulation of streamer propagation in air J. Comput. Phys. $2277222-45$

[42] Li C, Ebert U and Hundsdorfer W 2011 Simulated avalanche formation around streamers in an overvolted air gap IEEE Trans. Plasma Sci. 39 2256-7

[43] Zhelezniak M B, Mnatsakanian A K and Sizykh S V 1982 Photoionization of nitrogen and oxygen mixtures by radiation from a gas discharge Teplofizika Vysokikh Temp. 20 423-8

[44] Luque A and Gordillo-Vaquez F J 2011 Sprite beads originating from inhomogeneities in the mesospheric electron density Geophys. Res. Lett. 38 L04808

[45] Kossyi I A, Kostinsky A Y, Matveyev A A and Silakov V P 1992 Kinetic scheme of the non-equilibrium discharge in nitrogen-oxygen mixtures Plasma Sources Sci. Technol. 1207220

[46] Pancheshnyi S 2005 Role of electronegative gas admixtures in streamer start, propagation and branching phenomena Plasma Sources Sci. Technol. 14645653

[47] Pancheshnyi S 2009 personal communication

[48] Wannier G H 1953 Motion of gaseous ions in strong electric fields Bell Syst. Tech. J. 32170254

[49] Gordillo-Vzquez F J 2008 Air plasma kinetics under the influence of sprites J. Phys. D: Appl. Phys. 41234016

[50] Luque A and Gordillo-Vzquez F J 2011 Mesospheric electric breakdown and delayed sprite ignition caused by electron detachment Nat. Geosci. 52225

[51] Liu N 2012 Multiple ion species fluid modeling of sprite halos and the role of electron detachment of $\mathrm{O}$ in their dynamics J. Geophys. Res. 117 A03308 
[52] Popov N A 2010 Evolution of the negative ion composition in the afterglow of a streamer discharge in air Plasma Phys. Rep. 36812818

[53] Liu N, Kosar B, Sadighi S, Dwyer J R and Rassoul H K 2012 Formation of streamer discharges from an isolated ionization column at subbreakdown conditions Phys. Rev. Lett. 109025002

[54] Koppitz J 1973 Nitrogen discharges of large cross section at high overvoltage in a homogeneous field J. Phys. D: Appl. Phys. 6 1494-502

[55] Kunhardt E E 2000 Generation of large-volume, atmosphericpressure, nonequilibrium plasmas IEEE Trans. Plasma Sci. 28189200
[56] Palmer A J 1974 A physical model on the initiation of atmospheric-pressure glow discharges Appl. Phys. Lett. 25138

[57] Levatter J I and Lin S C 1980 Necessary conditions for the homogeneous formation of pulsed avalanche discharges at high gas pressures $J$. Appl. Phys. 51210

[58] Herziger G, Wollermann-Windgasse R and Banse K H 1981 On the homogeneization of transverse gas discharges by preionization Appl. Phys. 24267272

[59] Teunissen J, Sun A and Ebert U 2014 A time scale for electrical screening in pulsed gas discharges J. Phys. D: Appl. Phys. 47365203 